\title{
Investigating Differences in Nutritional Parameters in Ugandan Children with Plasmodium falciparum Severe Malaria
}

\author{
Lucy D. Brown ${ }^{1}$, Katrina $\mathrm{Co}^{2}$, Caitlin Bond ${ }^{2}$, Robert O. Opoka ${ }^{3}$, Dibyadyuti Datta ${ }^{2}$, Chandy C. \\ John², \\ ${ }^{1}$ Indiana University School of Medicine, Indianapolis, Indiana; \\ ${ }^{2}$ Ryan White Center for Pediatric Infectious Disease and Global Health, Department of \\ Pediatrics, Indiana University School of Medicine, Indianapolis, Indiana; \\ ${ }^{3}$ Department of Paediatrics and Child Health, Makerere University, Kampala, Uganda
}

Background: In 2018, malaria produced an estimated 272,000 deaths in children $<5$ years of age, accounting for $67 \%$ of all malaria deaths worldwide, with a majority in the WHO African Region. Malnutrition is an important risk factor for malaria. Wasting, Stunting and Underweight are crucial indicators of malnutrition. Annually, 14 million children $<5$ are classified as wasted and 59 million children are classified as stunted.

Objective: The objective of this study is to determine the association between each of the major manifestations of severe malaria (SM) and nutritional parameters - weight-for-age (WAZ), height-for-age (HAZ), and weight-for-height (WHZ) - in children from the Ugandan cities Mulago and Jinja.

Methods: To assess differences in WAZ, HAZ, and WHZ by the five types of SM and community controls (CC), we evaluated Z-scores from children $<5$ years old enrolled in a prospective cohort study (NDI, Neurodevelopmental Impairment in Children with Severe Malaria) at enrollment and 12-month follow-up.

Results: WAZ and WHZ at baseline were significantly lower among SM groups than in CC $(p<0.001)$, but there were no significant differences observed at 12-month follow-up. There were no major differences in HAZ between the SM and CC groups. WAZ and HAZ at baseline were significantly lower among High Mortality (HM) versus Low Mortality (LM) groups $(p<0.01)$. At 12month follow-up, HAZ remained significantly lower in HM versus LM $(p<0.01)$. There were no major differences in $\mathrm{WHZ}$ between the HM and LM groups. Compared to those who survived, the 27 children who died had significantly lower WAZ $(p<0.05)$, but no major differences in HAZ or WHZ.

Conclusion: Underweight, stunting, and wasting may be risk factors for SM. Overall, a more comprehensive understanding of how SM elicits adverse effects in children is necessary. Nutrition intervention programs must be implemented to prevent child stunting, wasting, underweight, and mortality. 